\title{
Article \\ Ozone Therapy for Early Childhood Caries (ECC) Treatment: An In Vivo Prospective Study
}

\author{
Valentina Luppieri ${ }^{1}$, Antonio Manfra ${ }^{2}$, Luca Ronfani ${ }^{1}\left(\mathbb{D}\right.$, Maddalena Chermetz $^{1}$ and Milena Cadenaro ${ }^{1,3, * \mathbb{C}}$ \\ 1 Institute for Maternal and Child Health—IRCCS “Burlo Garofolo"—Trieste, 34137 Trieste, Italy; \\ valentina.luppieri@burlo.trieste.it (V.L.); luca.ronfani@burlo.trieste.it (L.R.); \\ maddalena.chermetz@burlo.trieste.it (M.C.) \\ 2 Private Practice, 34100 Trieste, Italy; manfra.anto@gmail.com \\ 3 Department of Medical Sciences, University of Trieste, Strada di Fiume 447, 34149 Trieste, Italy \\ * Correspondence: milena.cadenaro@burlo.trieste.it
}

check for updates

Citation: Luppieri, V.; Manfra, A.; Ronfani, L.; Chermetz, M.; Cadenaro, M. Ozone Therapy for Early Childhood Caries (ECC) Treatment: An In Vivo Prospective Study. Appl. Sci. 2022, 12, 1964. https://doi.org/ 10.3390/app12041964

Academic Editor: Mary Anne Melo

Received: 19 January 2022

Accepted: 12 February 2022

Published: 14 February 2022

Publisher's Note: MDPI stays neutral with regard to jurisdictional claims in published maps and institutional affiliations.

Copyright: () 2022 by the authors. Licensee MDPI, Basel, Switzerland. This article is an open access article distributed under the terms and conditions of the Creative Commons Attribution (CC BY) license (https:/ / creativecommons.org/licenses/by/ $4.0 /)$.

\begin{abstract}
The present study aimed to evaluate the effectiveness of ozone therapy in (1) increasing the compactness of decayed dentin (main aim), (2) reducing dentin hypersensitivity, (3) decreasing the salivary bacterial count, and (4) improving the quality of life in ECC-affected children during a 3-month follow-up. A total of 20 subjects uncooperative with conventional restorative treatment were treated with a $60 \mathrm{~s}$ ozone application/week $\left(\mathrm{OzoneDTA}^{\circledR}\right)$ for 4 weeks. Patients were evaluated at $\mathrm{T} 0$ = baseline; $\mathrm{T} 1$ = after ozone cycle and T2, T3, T4 =1, 2, 3-month follow-up, respectively. Dentin compactness was evaluated according to the Affected Dentine Scale (ADS) proposed by the authors; dentin hypersensitivity was recorded with the Wong-Baker Faces Pain Rating Scale (WBFPRS); salivary bacteria were measured with the Saliva Check Mutans Test and quality of life with the Early Childhood Oral Health Impact Scale (ECOHIS). ADS values and salivary bacteria count results were analyzed using non-parametric tests. Statistically significant differences were detected for both parameters between T0 and T1 $(p<0.01)$. At T1, dentin hypersensitivity was resolved and quality of life improved. Ozone therapy is a simple and painless approach, which might be useful for controlling caries' progression in uncooperative children until appropriate cooperation is achieved.
\end{abstract}

Keywords: early childhood caries; ozone therapy; decayed dentin; dentin hypersensitivity; salivary bacterial count; quality of life

\section{Introduction}

Early Childhood Caries (ECC), once referred to as nursing bottle caries, milk bottle syndrome, or baby bottle tooth decay, is defined by the American Academy of Pediatric Dentistry (AAPD) as the presence of "one or more decayed (non-cavitated or cavitated lesions), missing (due to caries), or filled tooth surfaces in any primary tooth in a child of 71 months of age or younger" [1]. ECC is one of the chronic infectious childhood diseases that is most widespread worldwide, representing a major public health problem with a significant impact on society $[2,3]$. ECC prevalence varies widely among countries, reaching $70-85 \%$ in socioeconomically disadvantaged groups $[2,4,5] ; 3$ - to 4 -year-old boys are found to be more affected than girls [2].

ECC etiology is multifactorial and results from the interaction of cariogenic bacteria, i.e., Mutans streptococci, with fermentable carbohydrates on tooth surfaces over time [2,6]. The main risk factors for ECC development include a high salivary cariogenic bacterial load, a sugar-rich diet, improper oral hygiene habits, the presence of enamel defects, low family socioeconomic status, poor parental education, and a genetic predisposition [1-5].

Lesions start as whitish spots on the buccal surfaces of maxillary incisors (mild ECC) that may progress into cavitated defects involving also maxillary molars (moderate ECC) and mandibular teeth (severe ECC), leading to root stumps in the most critical cases [2]. 
Depending on the severity of the pathology, several significant consequences of ECC (pain, infections, etc.) may have a negative impact on a child's health and quality of life [2,6,7].

ECC management is complex and may require treatments under general anesthesia, especially in very young, severely affected children uncooperative with traditional dental treatments $[2,8-10]$.

Prevention and early diagnosis are essential to avoid the most frequent consequences related to ECC and allow a non-invasive or minimally invasive therapeutic approach, gaining patients' cooperation and reducing the care burden for both families and the National Health Service $[1,2,5,10]$.

Among minimally invasive treatments of ECC, ozone therapy is a health-safe, simple, painless, and ultra-conservative approach that might be useful for controlling caries progression in children uncooperative with traditional dental treatments until appropriate cooperation and compliance are achieved [11-14].

Thanks to its high oxidizing power, ozone $\left(\mathrm{O}_{3}\right)$ can oxidize the bacterial cell wall, causing its lysis, and the pyruvic acid produced by bacteria, turning it into acetic acid and carbon dioxide, arresting or reversing caries progression.

If the antimicrobial properties of ozone are well known [13,15-19], its remineralizing and desensitizing activities are controversial: by increasing the patency of dentinal tubules, ozone seems to enhance the diffusion of salivary ions to the surface of the decayed dentin, thus promoting its remineralization and reducing hypersensitivity [12-16,20-22].

The potential of the combined use of ozone with disinfectant agents, i.e., chlorhexidine (CHX), and remineralizing products i.e., sodium fluoride (NaF), nano-hydroxyapatite [13,23-26] and/or glass ionomer cements (GICs) [27], in halting or slowing down caries progression in ECC-affected children is positively described in the literature, while its efficacy when used alone, as well as its ideal concentration and the time needed to reach a full antimicrobial effect, remain controversial [13,23-27].

Given the data available so far, the present in vivo study aimed to deepen the knowledge on ozone therapy's effects in treating ECC-affected children uncooperative with traditional restorative treatments. The goal was to increase the decayed dentin's compactness and reduce the salivary bacterial count and dentin hypersensitivity, improving the patients' and their families' quality of life. The primary null hypothesis tested was that the use of ozone therapy on ECC-affected teeth would not modify the compactness of the affected dentin. The secondary null hypotheses tested were that ozone would not reduce the dentin hypersensitivity and salivary bacterial count and would not influence the quality of life in ECC-affected children during a 3-month follow-up.

\section{Materials and Methods}

\subsection{Study Design and Ethics Approval}

The present in vivo prospective observational study was carried out on 20 underage subjects, according to the principles expressed by the Helsinki Declaration, at the Pediatric Dental Clinic of the Institute for Maternal and Child Health-IRCCS "Burlo Garofolo"-Trieste, Italy, between January and December 2020. The Regional Ethics Committee (CEUR FVG) approved the study under the univocal code CEUR-2019-2595 Prot. N. 0008176/P/GEN/ARCS. The eligible children's parents/legal guardians were asked to provide written informed consent to the study prior to their enrolment.

\subsection{Study Objectives}

The primary aim of this study was to evaluate whether the in vivo treatment of ECCaffected teeth with ozone therapy was able to improve the decayed dentin's compactness at $\mathrm{T} 1$ (after the ozone therapy cycle) and T2, T3, and T4 (1, 2, and 3-month follow-up) in comparison to T0 (baseline) according to the Affected Dentine Scale (ADS), a clinical scale proposed by the authors. 
Secondary aims were to measure the dentin hypersensitivity of ECC-affected teeth according to the Wong-Baker Faces Pain Rating Scale (WBFPRS) at each follow-up; to record any changes in Streptococcus Mutans salivary load by using a specific salivary test, and to assess the patients' and their families' quality of life through the Early Childhood Oral Health Impact Scale (ECOHIS) between T0 and T1.

\subsection{Patient Recruitment}

Subjects were selected among children attending the Pediatric Dental Clinic of the Institute for Maternal and Child Health-IRCCS "Burlo Garofolo"-Trieste, Italy, according to the following inclusion and exclusion criteria.

Inclusion criteria: patients aged 71 months or younger, in good health conditions (i.e., no coexisting systemic diseases, no drug intake), presenting ECC (diagnosis formulated according to the AAPD definition [1]), not adequately cooperating with traditional restorative treatments.

Exclusion criteria: patients complaining of spontaneous pain or with abscesses/pulpitis; patients affected by epilepsy, serious neurological disorders, psychological disorders, severe asthma, carriers of cardiac stimulators (pacemakers), presenting excessive sensitivity to electric current or other conditions for which the use of the selected ozone medical device is contraindicated according to the manufacturer.

Each included patient was identified with a numeric ID code known by the researchers.

\subsection{Sample Size Calculation}

The sample size was calculated on the study's primary outcome (variation of the ADS score between $\mathrm{T} 0$ and T1), assuming a score variation of 1 point with a standard deviation of 1.5 points, setting beta at 0.2 (study power $80 \%$ ) and alpha at 0.05 . The minimum expected sample size required was 20 decayed teeth in 20 ECC-affected children (OpenEpi version 3, open-source calculator).

\subsection{Ozone Therapy}

Primary teeth affected by ECC were treated with an ozone therapy cycle, i.e., an ozone therapy session performed once a week for four consecutive weeks. The ozone generator used was the OzoneDTA ${ }^{\circledR}$ medical device (Sweden \& Martina) marked CE. OzoneDTA ${ }^{\circledR}$ is a small and portable device consisting of a control unit, a handpiece, a safety lever, a pedal, a power switching adapter, and a kit of 5 autoclavable probes with distinct terminal shapes, which can be used in different clinical conditions. The device has 10 selectable programs of different intensities.

The device was set on the intensity program $n^{\circ} 6$, as recommended by the manufacturer. The probe type was chosen depending on the tooth/teeth to be treated.

Teeth to be treated were cleaned of any plaque and food debris using a rotating brush mounted on a blue ring handpiece at low speed, isolated using cotton rolls and the saliva suction system, and dried with the dental air syringe (jet of compressed oil-free air). In each session, ozone was applied for $60 \mathrm{~s}$ per tooth, keeping the probe's tip in continuous motion and perpendicular to the decayed surface at $1 \mathrm{~mm}$ from it, as shown in Figure 1.

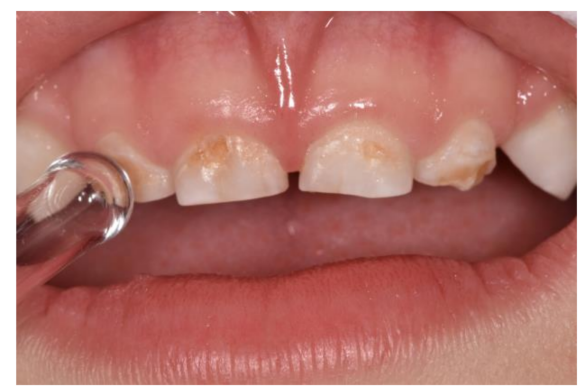

Figure 1. Probe $\mathrm{n}^{\circ} 3$ of the ozone device applied on upper primary incisors. 


\subsection{Clinical Evaluations}

Before performing any evaluation, patients and parents were educated and encouraged to perform proper at-home oral hygiene and maintain correct food habits.

Patients were examined on a recall schedule at the following times: $\mathrm{T} 0=$ baseline; $\mathrm{T} 1$ = after the ozone therapy cycle (at the end of the fourth ozone therapy session); T2 = 1-month follow-up (1 month from T1); T3 = 2-month follow-up (2 months from T1), and T4 $=3$-month follow-up (3 months from T1). In order to guarantee the consistency of the recordings throughout the study, clinical evaluations were performed by a single experienced examiner under optimal light conditions and on cleaned and dried teeth.

\subsubsection{Compactness of the Decayed Dentin}

The compactness of the decayed dentin was evaluated at each follow-up using a dental explorer and a dental excavator according to the Affected Dentin Scale (ADS), a clinical scale conceived by the authors. This scale classifies the decayed dentin according to four scores, as reported in Table 1.

An increment in the dentin's compactness corresponds to a reduction in the score and a slowdown of caries' activity.

Intra-examiner reliability was assessed using the kappa ( $\mathrm{k})$ test.

Table 1. Affected Dentin Scale (ADS) score.

\begin{tabular}{ll}
\hline $\mathbf{1}$ & Shiny, hard, blackish-brown decayed dentin \\
\hline $\mathbf{2}$ & Decayed dentin slightly explorable by the dental explorer's tip that produces slight grooves \\
\hline $\mathbf{3}$ & Decayed dentin explorable by the dental explorer's tip that produces marked grooves \\
\hline $\mathbf{4}$ & $\begin{array}{l}\text { Completely softened decayed dentin; the dental explorer penetrates the dentin, the excavator } \\
\text { removes it in layers }\end{array}$
\end{tabular}

\subsubsection{Dentin Hypersensitivity}

The ECC-related dentin hypersensitivity to thermal stimuli was evaluated at each follow-up using the dental unit air syringe (jet of compressed oil-free air spray at 45 psi pressure and environmental temperature of $19-24{ }^{\circ} \mathrm{C}$ applied for $2-3 \mathrm{~s}$ at a distance of $1 \mathrm{~cm}$ perpendicularly to the tooth surface) [28] and scored according to the Wong-Baker Faces Pain Rating Scale (WBFPRS) [29].

\subsubsection{Tooth Selection}

All primary teeth affected by ECC were treated with ozone for each patient. However, only a single ECC-affected tooth per patient, identified according to specific criteria, was subjected to dentin compactness and hypersensitivity evaluations at follow-ups. Selectable teeth had to present a carious lesion extended to no more than half of the tooth's crown and with an ADS score of 3 or 4 at baseline. If more than one tooth fulfilled these criteria, the most anterior was selected.

\subsubsection{Salivary Bacterial Count}

Streptococcus Mutans' salivary count was evaluated at T0 and T1 using the salivary test Saliva Check Mutans ${ }^{\circledR}$, GC Corporation, according to the protocol specified by the manufacturer. The test was considered positive if the patient's saliva contained more than 500.000 CFU of Streptococcus Mutans per milliliter.

Before the bacteria count, patients were instructed not to brush their teeth in the morning and avoid any food and drink for $1 \mathrm{~h}$ before the test.

\subsubsection{Quality of Life}

Patients' and families' ECC-related quality of life was evaluated through the Early Childhood Oral Health Impact Scale (ECOHIS) questionnaire, Italian validated version [30], submitted in an anonymous form to parents at T0 and T1. 
The questionnaire consisted of 13 questions addressed to patients' caregivers, divided into 5 main domains: patients' symptoms (question 1), daily life functions (questions 2-5), social and relationship life (questions 6-9), parents' emotional state (questions 10, 11), and economic aspects (questions 12,13). Each question had five possible answers depending on the frequency (never, hardly never, occasionally, often, very often), with a score from 0 to 4 points for a total of 52 points; 52 was the worst result while 0 was the best one.

Salivary tests and ECOHIS questionnaires were performed only at T0 and T1 to evaluate the direct effect of ozone therapy alone (one ozone therapy cycle), respectively, on patients' salivary bacterial load and on their quality of life.

\subsection{Statistical Analysis}

Statistical analysis was performed on the ADS scores and the salivary bacterial count results using non-parametric tests. The Friedman test was used to analyze the ADS values over time, while pairwise comparisons were made with the Wilcoxon test. The McNemar test was used to analyze the salivary bacterial count results (level of significance $p<0.01$ ).

Dentin hypersensitivity scores and ECOHIS questionnaires results were presented as descriptive analysis.

\section{Results}

A total of 20 subjects ( 9 males and 11 females) aged between 3 and 5 years (mean age 4.5) were enrolled in the study; a total of 20 teeth (one per patient: 9 molars, 8 incisors, and 1 canine) were considered for dentin compactness and dentin hypersensitivity clinical evaluations. No differences between boys and girls were found in the results.

\subsection{Clinical Evaluations}

\subsubsection{Compactness of the Decayed Dentin}

ADS values at each follow-up are reported in terms of means and standard deviation (SD) in Table 2; the trend of ADS mean values over time is illustrated in Figure 2.

The kappa test confirmed a high level of intra-examiner reliability $(\mathrm{k}=0.82)$. The Friedman test revealed a significant improvement in dentin compactness $(p=0.000)$ over time. The Wilcoxon test performed between time intervals revealed a statistically significant difference between T0 and T1 ( $p=0.000)$. Comparisons between other time points were not statistically significant (T1 and T2 $p=0.317$; T2 and T3 $p=1.000$; T3 and T4 $p=0.083$ ).

Table 2. ADS data shown as means \pm SD.

\begin{tabular}{ccccc}
\hline T0 & T1 & T2 & T3 & T4 \\
\hline $3.2 \pm 0.4$ & $2.1 \pm 0.5$ & $2.0 \pm 0.5$ & $2.0 \pm 0.5$ & $1.9 \pm 0.6$ \\
\hline
\end{tabular}

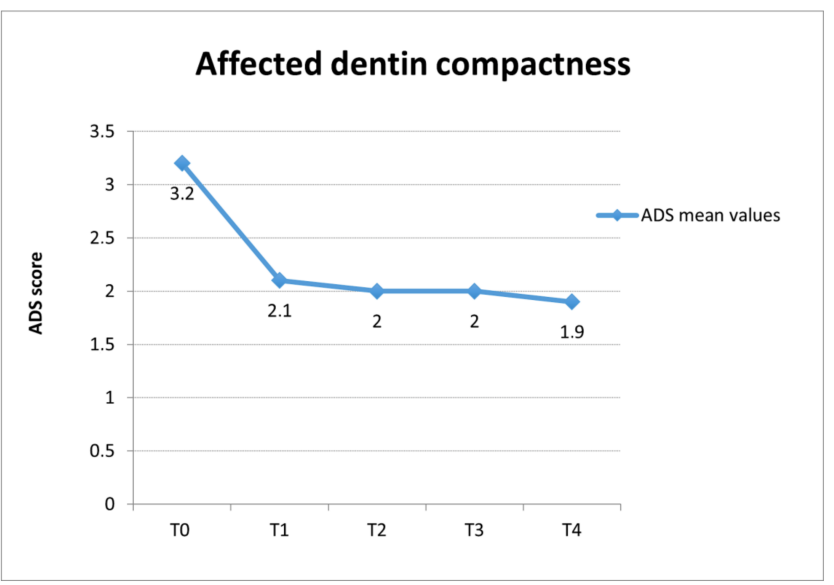

Figure 2. Trend of ADS mean scores over time (confidence level 95\%). 


\subsubsection{Dentin Hypersensitivity}

Only 2 among the 20 enrolled children referred to pain at the air-spray stimulation at T0, with a mean reported value of 3.5 according to the WBFPRS. At T1, none of the patients complained of dentin hypersensitivity in ECC-affected teeth; no changes were found at later follow-ups.

\subsubsection{Salivary Bacterial Count}

The salivary test was performed at T0 and T1 immediately after the last ozone application in 11 children out of 20 , since not all the enrolled children were sufficiently cooperative and able to spit their saliva correctly.

Nine of these 11 tests were positive for Streptococcus Mutans at T0, while two were negative. At T1, the bacteria count decreased significantly according to the McNemar test ( $p=0.008)$ : 8 of the 9 patients that were positive at T0 became negative at T1. In contrast, no changes were detected for the negative ones.

Salivary bacterial count results are illustrated as percentages in Figure 3.

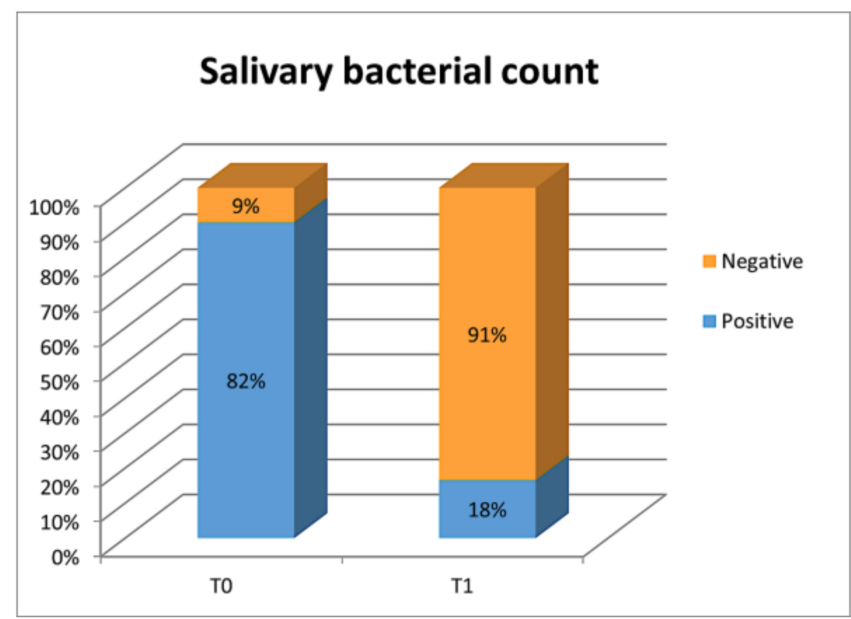

Figure 3. Salivary bacterial count.

\subsubsection{Quality of Life}

The results of the ECOHIS questionnaire are reported in Figure 4, which illustrates a comparison of the total score of each domain between T0 and T1. The mean ECOHIS score at T0 was 9.4, while that at T1 was 5.9.

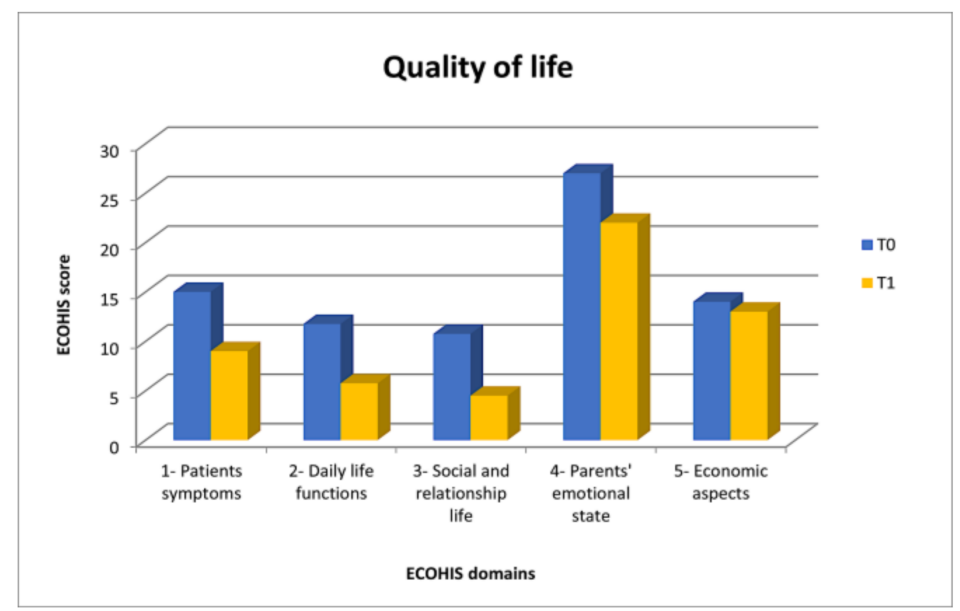

Figure 4. ECOHIS questionnaire results divided into domains. 


\section{Discussion}

Nowadays, ECC is one of the chronic infectious childhood diseases most widespread worldwide [2,3]. The potential of the combined use of ozone with disinfectant agents, i.e., chlorhexidine, and remineralizing products, i.e., sodium fluoride and/or nanohydroxyapatite [13,23-26] and/or glass ionomer cements (GICs) [27], in halting or slowing down caries progression in ECC-affected children is positively described in the literature, while its efficacy when used alone, as well as its ideal concentration and the time needed to reach a full antimicrobial effect, remain controversial [13,23-27].

Ozone is an oxidizing agent whose activity is selective on pathogens and has no disadvantages or negative effects on human body cells [15-18]. Its well-known disinfectant and biostimulating properties are used in several medical fields, e.g., in chronic inflammatory disease management, thanks to its ability to activate the immune system and the antihypoxic and biosynthetic biological processes [15-18]. In dentistry, ozone has several applications in dental caries and hypersensitivity treatment, the acceleration of mucosal healing, and the disinfection of root canals [15-18].

The rationale of this study was that ozone, thanks to its bactericidal and analgesic properties [13,15-19], improving the decayed dentin compactness and decreasing the salivary bacterial count, might arrest or slow down caries progression, reducing dentin hypersensitivity and thus improving patients' and families' quality of life.

The lack of an evident gender prevalence among the enrolled patients ( 9 males vs. 11 females) contrasted with literature data reporting that boys are more affected than girls [2].

Data about the decayed dentin's compactness showed a significant decrease from T0 (mean value 3.2), when dentin was explorable by the dental explorer's tip, which produced marked grooves, to T1 (mean value 2.1), when it was only slightly explorable according to the ADS score description, remaining stable at the subsequent follow-ups. Thus, the primary null hypothesis tested was rejected. The increase in the decayed dentin's compactness between $\mathrm{T} 0$ and $\mathrm{T} 1$ may be explained by the ozone's oxidizing power, which can slow down the demineralization process and encourage dentin remineralization by inhibiting cariogenic bacteria and stimulating the production of a sound reactive dentinal barrier [12-20]. The maintenance of this improvement over time can be explained considering the stimulating effect of ozone on the formation of reactive dentin [12-20].

However, it is essential to specify that the advice given to families about at-home daily oral hygiene and diet may have biased the results, helping to improve the compactness of the treated decayed dentin over time. The authors should not have provided any advice to avoid this bias, but it would have been unethical.

Ozone therapy also helped to reduce the ECC-related dentin hypersensitivity when present, leading to the rejection of the second null hypothesis. At T0, only 2 of the 20 enrolled patients complained of dentin hypersensitivity to compressed air spray (average value 3.5 measured according to the WBFPRS), and none of them complained about spontaneous symptoms. After the ozone therapy cycle, dentin hypersensitivity was resolved entirely and the improvement remained stable over time. According to the hydrodynamic theory, dentin hypersensitivity is due to the fluids' movement into the dentinal tubules, caused by external stimuli that stimulate the pulp pain receptors [31]. In this context, ozone can neutralize the acidic proteins produced by cariogenic bacteria, representing the osmotic stimulus responsible for the movement of fluids in dentinal tubules that leads to hypersensitivity [20-22]. It has to be reported that recording patients dentin hypersensitivity was not always easy since they were not always able to provide reliable feedback due to their young age.

Encouraging results were also obtained from salivary tests that assessed the presence of Streptococcus Mutans, the primary bacterium involved in the onset of the carious process [32,33]. At T0, 82\% of the tested patients were positive, i.e., their Streptococcus Mutans salivary count was higher than $500.000 \mathrm{CFU} / \mathrm{mL}$, and these results were compatible with their clinical condition of multiple caries in an active phase. At T1, results were almost 
the opposite of those at T0 and only 9\% of the patients were positive for Streptococcus Mutans; thus, the third null hypothesis was rejected. This significant result can be explained thanks to both the antimicrobial activity of ozone [13,15-17] and the improvement of the patients' oral hygiene and food habits, which, however, might also represent a bias in the evaluation of ozone antibacterial effects.

Moreover, an important result was also gained in patients' and their family's quality of life and so the null hypothesis that ozone therapy on ECC-affected teeth would not alter the quality of the affected dentin was also rejected.

The results of ECOHIS questionnaires showed that dental caries had a negative impact on the quality of life for both patients and their families; from this point of view, ECC is, in fact, not only an oral problem but it might also have systemic consequences and psychological and economic repercussions [2,6,7].

For each of the five main domains of the questionnaire, a reduction in the scores was registered between $\mathrm{T} 0$ and $\mathrm{T} 1$. The total scores of the pain symptoms (domain 1) decreased by six points (15 to 9), showing the efficacy of ozone analgesic activity [19-22]. Comparing the answers given by parents to the first question of the questionnaire (domain 1) and those given by patients about their perceived dentin hypersensitivity evaluated at the dental chair according to the WBFPRS, an inconsistency is evident. This might be explained considering preschool children's difficulty in expressing their symptoms appropriately due to their young age. The decrease in the scores for the answers to the questions on oral functions such as nutrition and language (domain 2; mean score 11.75-10.75), as well as of those on social life and relations (domain 3; mean score 5.75-4.5), showed an essential improvement in these conditions and a positive impact of ozone therapy also on the psychological and social spheres of patients and their families. ECC had, in fact, a substantial impact not only on affected children but also on their families, and this was highly appreciable in the answers to questions on the patient's caregivers' emotional state and economic and financial aspects (domains 4 and 5). Most parents stated that they felt upset and guilty for their children's oral status and declared that they had to take days off from work to take them to the dentist. Even if the scores of these domains decreased from T0 to T1 (mean scores 27-22 and 14-13, respectively), they remained higher compared to the other domains. This is probably because parents had to take their children to the dental appointments scheduled for this study. Furthermore, at T1, parents were more aware of ECC and its consequences, being also more conscious of the importance of oral health for systemic wellness [2,6,7].

Salivary tests and ECOHIS questionnaires were performed at T0 and T1 to evaluate the direct effect of ozone therapy on patients' salivary bacterial load and their quality of life immediately after treatment.

Ozone therapy has several advantages: being a simple and non-invasive technique, it can be easily used in young and/or anxious or fearful pediatric patients to improve their cooperation and help them to become used to traditional restorative treatments [14]. In addition, being a non-aerosol-producing therapy, it is especially recommended during this unique COVID-19 pandemic time because it helps reduce the risk of cross-infections in the dental practice [34].

The main limitations of ozone therapy depend on the fact that it is not indicated if patients complain about spontaneous pain such as pulpitis or present abscesses, and families have also to be cooperative since the ozone cycle requires several scheduled dental sessions in a short period (four ozone therapy sessions performed once a week for four consecutive weeks).

As this was a preliminary study, further research evaluating the effects of ozone therapy on a higher number of ECC-affected teeth, for a longer follow-up period, and also in comparison with other treatments, i.e., remineralizing strategies such as the application of fluoride varnishes, non-fluoride remineralizing agents such as calcium phosphate (CPP$\mathrm{ACP}$ ), and/or innovative biomimetic remineralization systems, e.g., those based on $\mathrm{Zn}$ - 
carbonate hydroxyapatite $\left[\mathrm{Ca}_{5}\left(\mathrm{PO}_{4}\right)_{3} \mathrm{OH}\right][13,23,24,35]$, are needed to obtain more complete results on this topic.

\title{
5. Conclusions
}

Based on the obtained results, ozone therapy may be considered an effective technique for treating ECC-affected teeth in preschool children uncooperative with traditional restorative treatments since it helps to slow down the caries progression and improve patients' quality of life. Moreover, being a simple and non-invasive technique, it may help children to become used to dental treatments.

\begin{abstract}
Author Contributions: Conceptualization, M.C. (Maddalena Chermetz) and M.C. (Milena Cadenaro); methodology, M.C. (Maddalena Chermetz), L.R. and M.C. (Milena Cadenaro); investigation, A.M.; resources, V.L., A.M., L.R. and M.C. (Maddalena Chermetz); data curation, A.M. and M.C. (Maddalena Chermetz); writing-original draft preparation, V.L. and M.C. (Maddalena Chermetz); writingreview and editing, M.C. (Milena Cadenaro); visualization, V.L., A.M., L.R., M.C. (Maddalena Chermetz) and M.C. (Milena Cadenaro); supervision, L.R. and M.C. (Milena Cadenaro). All authors have read and agreed to the published version of the manuscript.
\end{abstract}

Funding: This research received no external funding.

Institutional Review Board Statement: The study was conducted according to the guidelines of the Declaration of Helsinki and approved by the Friuli Venezia Giulia Ethics Committee (CEUR FVG) under the univocal code CEUR-2019-2595 Prot. N. 0008176/P/GEN/ARCS.

Informed Consent Statement: Informed consent was obtained from all the parents or legal guardians of the included subjects.

Data Availability Statement: The data presented in this study are available on request from the corresponding author. The data are not publicly available due to privacy restrictions.

Conflicts of Interest: The authors declare no conflict of interest.

\section{References}

1. American Academy of Pediatrics. Policy on Early Childhood Caries (ECC): Classifications, Consequences, and Preventive Strategies. In The Reference Manual of Pediatric Dentistry; American Academy of Pediatrics: Itasca, IL, USA, 2020 ; pp. 79-81.

2. Anil, S.; Anand, P.S. Early Childhood Caries: Prevalence, Risk Factors, and Prevention. Front. Pediatr. 2017, 5, 157. [CrossRef]

3. Kirthiga, M.; Murugan, M.; Saikia, A.; Kirubakaran, R. Risk Factors for Early Childhood Caries: A Systematic Review and Meta-Analysis of Case Control and Cohort Studies. Pediatr. Dent. 2019, 41, 95-112. [PubMed]

4. Congiu, G.; Campus, G.; Lugliè, P.F. Early Childhood Caries (ECC) Prevalence and Background Factors: A Review. Oral Health Prev. Dent. 2014, 12, 71-76. [CrossRef] [PubMed]

5. Colombo, S.; Gallus, S.; Beretta, M.; Lugo, A.; Scaglioni, S.; Colombo, P.; Paglia, M.; Gatto, R.; Marzo, G.; Caruso, S.; et al Prevalence and Determinants of Early Childhood Caries in Italy. Eur. J. Paediatr. Dent. 2019, 20, 267-273. [CrossRef] [PubMed]

6. Nunn, M.E.; Braunstein, N.S.; Krall Kaye, E.A.; Dietrich, T.; Garcia, R.I.; Henshaw, M.M. Healthy Eating Index Is a Predictor of Early Childhood Caries. J. Dent. Res. 2009, 88, 361-366. [CrossRef]

7. Collado, V.; Pichot, H.; Delfosse, C.; Eschevins, C.; Nicolas, E.; Hennequin, M. Impact of Early Childhood Caries and Its Treatment under General Anesthesia on Orofacial Function and Quality of Life: A Prospective Comparative Study. Med. Oral Patol. Oral Cirugia Bucal 2017, 22, e333-e341. [CrossRef]

8. Zaror, C.; Matamala-Santander, A.; Ferrer, M.; Rivera-Mendoza, F.; Espinoza-Espinoza, G.; Martínez-Zapata, M.J. Impact of Early Childhood Caries on Oral Health-Related Quality of Life: A Systematic Review and Meta-Analysis. Int. J. Dent. Hyg. 2022, 20, 120-135. [CrossRef]

9. Duangthip, D.; Jiang, M.; Chu, C.H.; Lo, E.C.M. Restorative Approaches to Treat Dentin Caries in Preschool Children: Systematic Review. Eur. J. Paediatr. Dent. 2016, 17, 113-121.

10. Phantumvanit, P.; Makino, Y.; Ogawa, H.; Rugg-Gunn, A.; Moynihan, P.; Petersen, P.E.; Evans, W.; Feldens, C.A.; Lo, E.; Khoshnevisan, M.H.; et al. WHO Global Consultation on Public Health Intervention against Early Childhood Caries. Community Dent. Oral Epidemiol. 2018, 46, 280-287. [CrossRef]

11. Duangthip, D.; Jiang, M.; Chu, C.H.; Lo, E.C.M. Non-Surgical Treatment of Dentin Caries in Preschool Children-Systematic Review. BMC Oral Health 2015, 15, 44. [CrossRef]

12. Almaz, M.E.; Sönmez, I.Ş. Ozone Therapy in the Management and Prevention of Caries. J. Formos. Med. Assoc. Taiwan Yi Zhi 2015, 114, 3-11. [CrossRef] 
13. Ximenes, M.; Cardoso, M.; Astorga, F.; Arnold, R.; Pimenta, L.A.; Viera, R.D.S. Antimicrobial Activity of Ozone and NaFChlorhexidine on Early Childhood Caries. Braz. Oral Res. 2017, 31, e2. [CrossRef]

14. Dähnhardt, J.E.; Jaeggi, T.; Lussi, A. Treating Open Carious Lesions in Anxious Children with Ozone. A Prospective Controlled Clinical Study. Am. J. Dent. 2006, 19, 267-270.

15. Suh, Y.; Patel, S.; Kaitlyn, R.; Gandhi, J.; Joshi, G.; Smith, N.L.; Khan, S.A. Clinical Utility of Ozone Therapy in Dental and Oral Medicine. Med. Gas Res. 2019, 9, 163-167. [CrossRef]

16. Sen, S.; Sen, S. Ozone Therapy a New Vista in Dentistry: Integrated Review. Med. Gas Res. 2020, 10, 189-192. [CrossRef]

17. Elvis, A.M.; Ekta, J.S. Ozone Therapy: A Clinical Review. J. Nat. Sci. Biol. Med. 2011, 2, 66-70. [CrossRef]

18. Viebahn-Haensler, R.; León Fernández, O.S. Ozone in Medicine. The Low-Dose Ozone Concept and Its Basic Biochemical Mechanisms of Action in Chronic Inflammatory Diseases. Int. J. Mol. Sci. 2021, 22, 7890. [CrossRef]

19. Santos, G.M.; Pacheco, R.L.; Bussadori, S.K.; Santos, E.M.; Riera, R.; de Oliveira Cruz Latorraca, C.; Mota, P.; Benavent Caldas Bellotto, E.F.; Martimbianco, A.L.C. Effectiveness and Safety of Ozone Therapy in Dental Caries Treatment: Systematic Review and Meta-Analysis. J. Evid.-Based Dent. Pract. 2020, 20, 101472. [CrossRef]

20. Azarpazhooh, A.; Limeback, H.; Lawrence, H.P.; Fillery, E.D. Evaluating the Effect of an Ozone Delivery System on the Reversal of Dentin Hypersensitivity: A Randomized, Double-Blinded Clinical Trial. J. Endod. 2009, 35, 1-9. [CrossRef]

21. Lena, K.; Marianne, K. Ozone Treatment on Dentin Hypersensitivity Surfaces-A Pilot Study. Open Dent. J. 2017, 11, 65-70. [CrossRef]

22. Raafat Abdelaziz, R.; Mosallam, R.S.; Yousry, M.M. Tubular Occlusion of Simulated Hypersensitive Dentin by the Combined Use of Ozone and Desensitizing Agents. Acta Odontol. Scand. 2011, 69, 395-400. [CrossRef]

23. Johansson, E.; van Dijken, J.W.V.; Karlsson, L.; Andersson-Wenckert, I. Treatment Effect of Ozone and Fluoride Varnish Application on Occlusal Caries in Primary Molars: A 12-Month Study. Clin. Oral Investig. 2014, 18, 1785-1792. [CrossRef]

24. Atabek, D.; Oztas, N. Effectiveness of Ozone with or without the Additional Use of Remineralizing Solution on Non-Cavitated Fissure Carious Lesions in Permanent Molars. Eur. J. Dent. 2011, 5, 393-399. [CrossRef]

25. Hauser-Gerspach, I.; Pfäffli-Savtchenko, V.; Dähnhardt, J.E.; Meyer, J.; Lussi, A. Comparison of the Immediate Effects of Gaseous Ozone and Chlorhexidine Gel on Bacteria in Cavitated Carious Lesions in Children in Vivo. Clin. Oral Investig. 2009, 13, 287-291. [CrossRef]

26. Grocholewicz, K.; Matkowska-Cichocka, G.; Makowiecki, P.; Droździk, A.; Ey-Chmielewska, H.; Dziewulska, A.; Tomasik, M.; Trybek, G.; Janiszewska-Olszowska, J. Effect of Nano-Hydroxyapatite and Ozone on Approximal Initial Caries: A Randomized Clinical Trial. Sci. Rep. 2020, 10, 11192. [CrossRef]

27. Beretta, M.; Federici Canova, F. A New Method for Deep Caries Treatment in Primary Teeth Using Ozone: A Retrospective Study. Eur. J. Paediatr. Dent. 2017, 18, 111-115. [CrossRef]

28. Raposo, F.; de Carvalho Rodrigues, A.C.; Lia, É.N.; Leal, S.C. Prevalence of Hypersensitivity in Teeth Affected by Molar-Incisor Hypomineralization (MIH). Caries Res. 2019, 53, 424-430. [CrossRef]

29. Birnie, K.A.; Hundert, A.S.; Lalloo, C.; Nguyen, C.; Stinson, J.N. Recommendations for Selection of Self-Report Pain Intensity Measures in Children and Adolescents: A Systematic Review and Quality Assessment of Measurement Properties. Pain 2019, 160, 5-18. [CrossRef]

30. Contaldo, M.; Della Vella, F.; Raimondo, E.; Minervini, G.; Buljubasic, M.; Ogodescu, A.; Sinescu, C.; Serpico, R. Early Childhood Oral Health Impact Scale (ECOHIS): Literature Review and Italian Validation. Int. J. Dent. Hyg. 2020, 18, 396-402. [CrossRef]

31. Liu, X.-X.; Tenenbaum, H.C.; Wilder, R.S.; Quock, R.; Hewlett, E.R.; Ren, Y.-F. Pathogenesis, Diagnosis and Management of Dentin Hypersensitivity: An Evidence-Based Overview for Dental Practitioners. BMC Oral Health 2020, 20, 220. [CrossRef]

32. Valdez, R.M.A.; Duque, C.; Caiaffa, K.S.; Dos Santos, V.R.; de Loesch, M.L.A.; Colombo, N.H.; Arthur, R.A.; de Negrini, T.C.; Boriollo, M.F.G.; Delbem, A.C.B. Genotypic Diversity and Phenotypic Traits of Streptococcus Mutans Isolates and Their Relation to Severity of Early Childhood Caries. BMC Oral Health 2017, 17, 115. [CrossRef] [PubMed]

33. Ravikumar, D.; Ramani, P.; Gayathri, R. Genotypic Diversity of Streptococcus Mutans in Children with and without Early Childhood Caries- A Systematic Review. J. Oral Biol. Craniofacial Res. 2021, 11, 308-312. [CrossRef] [PubMed]

34. Paglia, L. COVID-19 and Paediatric Dentistry after the Lockdown. Eur. J. Paediatr. Dent. 2020, 21, 89. [CrossRef] [PubMed]

35. Butera, A.; Pascadopoli, M.; Gallo, S.; Lelli, M.; Tarterini, F.; Giglia, F.; Scribante, A. SEM/EDS Evaluation of the Mineral Deposition on a Polymeric Composite Resin of a Toothpaste Containing Biomimetic Zn-Carbonate Hydroxyapatite (MicroRepair ${ }^{\circledR}$ ) in Oral Environment: A Randomized Clinical Trial. Polymers 2021, 13, 2740. [CrossRef] 